\title{
Erstes Jahrestreffen der Internationalen Gesellschaft zur Prävention tabakinduzierter Erkrankungen (PTID)
}

\author{
E. Heissen \\ Radioonkologie und Nuklearmedizin, Ev. Krankenhaus Mülheim, Germany
}

Am 28.10.2002 fand im Kurssaal des Auditorium maximum der Universitätsklinik Essen das erste Jahrestreffen der «International Society for the Prevention of Tobacco Induced Diseases - PTID» statt. Es war verbunden mit einer wissenschaftlichen Tagung mit Teilnehmern aus Kanada, England, Hongkong, Japan, Polen und den USA. Deren Vorträge beschäftigten sich unter anderem mit einem Tiermodell bezüglich der akuten Pankreatitis nach Nikotinexposition, der Analyse von Tumormarkern bei Rauchern und Nichtrauchern, dem Rauchen als einem Faktor in der Entwicklung einer Medikamentenresistenz bei der Tuberkulose, der Wirksamkeit von Rauchverboten am Arbeitsplatz, dem Rückgang der Sterblichkeit an koronaren Herzkrankheiten in Folge der Reduktion des Nikotinabusus, der Wirksamkeit und Sicherheit einer Nikotinersatztherapie während der Schwangerschaft, den Gesundheitsrisiken durch Passivrauchen und einem niedrigen Geburtsgewicht als Hinweis auf Rauchen während der Schwangerschaft. Der Gründer und erste Präsident der Gesellschaft, Prof. Ed Nelson, Toxikologe am Universitätsklinikum Essen, war leider am 11.7.2002 verstorben. Er hatte bereits am 24.8.1998 ein wissenschaftliches Symposium «Passive Smoking and Pregnancy» veranstaltet. Dr. David A. Scott von der Universität Manitoba in Kanada folgt Ed Nelson als Präsident der PTID, als sein Stellvertreter wurde Prof. Anthony J. Hedley vom Medical Center der Universität Hongkong gewählt. Schatzmeister ist weiterhin Dr. Wojciech Hanke vom Nofer Institut für Arbeitsmedizin in Lodz, Polen. Das nächste Treffen wird vom 29.9. bis zum 1.10.2003 in Winnipeg, Kanada, stattfinden. Weitere Informationen und alle Einzelheiten über die Konferenz-Website www.umanitoba.ca/faculties/ dentistry/oral_biology/research/conference.

Die PTID ist eine nicht gewinnorientierte, akademische, wissenschaftliche, humanitäre Organisation von im Gesundheitswesen beruflich Tätigen, unabhängig von irgendeiner industriellen, politischen, regierungsamtlichen oder ideologischen
Gruppierung. Die Mitgliedschaft steht allen im Gesundheitswesen beruflich Beschäftigten offen. Es gibt aktive, unterstützende und Ehrenmitglieder. Jedes aktive Mitglied sollte einen akademischen Grad einer Universität oder eines College im medizinischen, zahnmedizinischen, biomedizinischen, biologischen, chemischen, sozialen oder psychologischen Bereich, der Rechtswissenschaften oder anderen relevanten wissenschaftlichen Gebieten haben. Es sollte aktiv beteiligt oder zumindest stark engagiert sein in klinischer und Grundlagenforschung und/oder der Lehre auf einem tabakbezogenen Gebiet. Es darf nicht direkt oder indirekt mit der Tabakindustrie verbunden sein. Die Tabakindustrie ist definiert als jede Gruppe, Organisation oder direkt dort beschäftigte Einzelperson, ebenso solche, die Tabak oder Tabakprodukte herstellen, vertreiben, verkaufen oder propagieren. Unterstützende Mitglieder können Schulen, Colleges, Universitäten, Forschungseinheiten, Kliniken, Krankenhäuser und Gesundheitsstiftungen sein, auch medizinische Gesellschaften, Journalisten, Versicherungsgesellschaften oder nicht der Tabakindustrie zugehörige Firmen. Die Aufnahmegebühr beträgt 10,- $€$, der Jahresbeitrag 50,- $€$, für Studierende 20,- $€$.«Tobacco Induced Diseases» ist die offizielle, internationale, «peer-reviewed» Zeitschrift der Gesellschaft, herausgegeben von Dr. Daniel Longo von der Universität Missouri, Columbia. Sie wird von der Sektion Öffentlichkeitsarbeit der Gesellschaft selbst produziert und erscheint vierteljährlich. Ihre Aufgabe ist die schnelle Veröffentlichung von original-wissenschaftlichen Arbeiten in der tabakbezogenen Forschung: Sozialwissenschaften, Epidemiologie, Krebs, Kardiologie, Toxikologie, Uro-Nephrologie, Gynäkologie usw. Der jährliche Subskriptionspreis beläuft sich auf 30,- $€$ für Studierende, 50,- $€$ für aktive PTIDMitglieder, 125,- $€$ für Nichtmitglieder und 250,- $€$ für Institutionen.

Weitere Informationen über die Gesellschaft, eine Mitgliedschaft und die Zeitschrift unter www.ptid.org.

\begin{tabular}{ll}
\hline KARGER & @ 2003 S. Karger GmbH, Freiburg \\
$\begin{array}{l}\text { Fax +497614520714 } \\
\begin{array}{l}\text { E-mail Information@Karger.de } \\
\text { www.karger.com }\end{array}\end{array}$ & $\begin{array}{l}\text { Accessible online at: } \\
\text { www.karger.com/onk }\end{array}$
\end{tabular}

